\title{
SPA-Resistant Scalar Multiplication on Hyperelliptic Curve Cryptosystems Combining Divisor Decomposition Technique and Joint Regular Form
}

\author{
Toru Akishita, Masanobu Katagi, and Izuru Kitamura \\ Information Technologies Laboratories, Sony Corporation, \\ 6-7-35 Kitashinagawa, Shinagawa-ku, Tokyo, 141-0001 Japan \\ akishita@pal.arch.sony.co.jp, \\ \{Masanobu.Katagi, Izuru.Kitamura\}@jp.sony.com
}

\begin{abstract}
Hyperelliptic Curve Cryptosystems (HECC) are competitive to elliptic curve cryptosystems in performance and security. Recently efficient scalar multiplication techniques using a theta divisor have been proposed. Their application, however, is limited to the case when a theta divisor is used for the base point. In this paper we propose efficient and secure scalar multiplication of a general divisor for genus 2 HECC over $\mathbb{F}_{2^{m}}$. The proposed method is based on two novel techniques. One is divisor decomposition technique in which a general divisor is decomposed into two theta divisors. The other is joint regular form for a pair of integers that enables efficient and secure simultaneous scalar multiplication of two theta divisors. The marriage of the above two techniques achieves both about $19 \%$ improvement of efficiency compared to the standard method and resistance against simple power analysis without any dummy operation.
\end{abstract}

Keywords: hyperelliptic curve cryptosystems, scalar multiplication, theta divisor, signed binary representation, simple power analysis.

\section{Introduction}

Elliptic Curve Cryptosystems (ECC) have increased their importance in public key cryptosystems because of their higher efficiency than RSA cryptosystems. Hyperelliptic Curve Cryptosystems (HECC) are generalization of ECC: ECC just correspond to HECC of genus 1. The security of HECC whose genus is smaller than 4 is thought to match that of ECC of the same group size. On the other side, the performance of HECC was believed to be slower than that of ECC due to their complex group operations. However, since Harley proposed an efficient group addition and doubling algorithm, so-called Harley algorithm, for genus 2 curves of odd characteristics in 2000 [7, optimizations and generalizations of Harley algorithm have been carried out 2, and at present HECC are competitive to ECC also in performance. 
In recent years, a new class of attacks has been proposed to extract some secret information from a cryptographic device using its power consumption: socalled power analysis. This paper deals with only Simple Power Analysis (SPA), which utilizes a power consumption trace during a single execution. Differential Power Analysis (DPA) is the more sophisticated attack that requires many power consumption traces with statistical tools.

In regard to HECC, the countermeasure against SPA must be considered when an ephemeral and secret scalar is used for scalar multiplication. The standard countermeasure is the double-and-add-always method that always repeats a divisor class doubling and a divisor class addition per bit of the scalar 3 . Recently the useful countermeasure for ECC, Montgomery ladder, was applied to HECC [4, but underlying curves of the HECC version are limited.

On the contrary, efficient and SPA-resistant scalar multiplication techniques peculiar to HECC have been recently proposed, which use a theta divisor 98. A theta divisor has weight smaller than the genus of the underlying curve. For a genus 2 hyperelliptic curve over $\mathbb{F}_{q}$, a theta divisor is represented as $D=(x+$ $\left.u_{0}, v_{0}\right)$, whereas a general divisor is represented as $D=\left(x^{2}+u_{1} x+u_{0}, v_{1} x+v_{0}\right)$, where $u_{i}, v_{i} \in \mathbb{F}_{q}$. The cost of an addition of a theta divisor is smaller than that of a general divisor due to its simple representation, so that scalar multiplication of a theta divisor is faster than that of a general divisor. This efficiency, however, can be utilized in the limited case, when the base point is a theta divisor and scalar multiplication of the base point is carried out, for example, in HEC DiffieHellman phase 1 or HEC-DSA signature generation.

In this paper we enhance the efficient use of a theta divisor to scalar multiplication of a general divisor for a genus 2 curve over $\mathbb{F}_{2^{m}}$. The enhancement is based on the following two novel techniques. The first one is Divisor Decomposition Technique (DDT). A general divisor $D=(U(x), V(x))$ can be decomposed into two theta divisors $D_{1}$ and $D_{2}$ if $U(x)$ is reducible over $\mathbb{F}_{2^{m}}$. The second one is Joint Regular Form (JRF), which is a new signed binary representation of a pair of integers such that one is even and the other is odd. Any signed bits at the same position of JRF satisfy that one is 0 and the other is \pm 1 .

In order to utilize both DDT and JRF, we compute $d D_{1}+(d+1) D_{2}$ and then subtract $D_{2}$ as compensation instead of the scalar multiplication $d D$. The simultaneous scalar multiplication $d D_{1}+(d+1) D_{2}$ with JRF of $(d, d+1)$ repeats a divisor class doubling and an addition of a theta divisor $\pm D_{1}$ or $\pm D_{2}$ per bit of $d$. Its cost is almost equal to the cost of the double-and-add-always method of a theta divisor, and is smaller than that of a general divisor. Moreover, SPAresistance is guaranteed because of regularity without any dummy operation, which causes the possibility of fault-based attacks. Even if $D$ is unable to be decomposed into $D_{1}$ and $D_{2}$, we update $D$ by repeating a divisor class doubling of $D$ until $D$ can be decomposed into $D_{1}$ and $D_{2}$. Then, after computing $\left(d D_{1}+\right.$ $\left.(d+1) D_{2}\right)-D_{2}=d D$, we repeat a divisor class halving [10] of $d D$ the corresponding times. The proposed method is $18.7 \%$ faster than the double-and-add-always method of a general divisor. 
The rest of paper is organized as follows. In next two sections, we briefly introduce HECC mainly focused to theta divisors and scalar multiplication. In Section 4 and 5, we present two novel techniques: Divisor Decomposition Technique (DDT) and Joint Regular Form (JRF). In Section 6, we show the efficient and secure scalar multiplication of a general divisor by combining DDT and JRF. Section 7 analyzes the computational efficiency of the proposed method. Finally, we draw our conclusion and discuss further work in Section 8 .

\section{Hyperelliptic Curve Cryptosystems}

We give only a brief introduction of Hyperelliptic Curve Cryptosystems (HECC) because of space limitation. More details can be found, for example, in 1211.

In this paper, we discuss genus 2 HECC over $\mathbb{F}_{2^{m}}$. A hyperelliptic curve over $\mathbb{F}_{2^{m}}$ is defined by $C: y^{2}+h(x) y=f(x)$, where $h(x)=x^{2}+h_{1} x+h_{0} \in \mathbb{F}_{2^{m}}[x]$ and $f(x)=x^{5}+\sum_{i=0}^{3} f_{i} x^{i} \in \mathbb{F}_{2^{m}}[x]$. In contrast to ECC, points $P$ on a hyperelliptic curve $C$ do not form a group. The group law is defined over Jacobian variety $\mathcal{J}_{C}$. $\mathcal{J}_{C}$ is isomorphic to a divisor class group which forms an additive group, and each divisor class is uniquely represented as a reduced divisor. A reduced divisor $D=\sum m_{i} P_{i}-\left(\sum m_{i}\right) P_{\infty}$, where $P_{i}=\left(x_{i}, y_{i}\right)$ and $P_{\infty}$ is a point at infinity, can be represented by two polynomials $(U(x), V(x))$ [16],

$$
\begin{gathered}
U(x)=\prod_{i}\left(x+x_{i}\right)^{m_{i}}, \quad V\left(x_{i}\right)=y_{i}, \\
\operatorname{deg} V<\operatorname{deg} U \leq 2, \quad V^{2}+h V+f \equiv 0 \bmod U .
\end{gathered}
$$

The $\operatorname{deg} U$ of a reduced divisor is called weight. We denote the weight of a reduced divisor $D$ by $w(D)$.

\subsection{Theta Divisor and General Divisor}

In the case of genus 2 hyperelliptic curves, a reduced divisor has weight smaller than or equal to 2. A divisor is called a theta divison 1 if its weight is smaller than 2 12. On the other hand, we call a divisor of weight 2 a general divisor. A theta divisor is represented as $D=\left(x+u_{0}, v_{0}\right)$, whereas a general divisor is represented as $D=\left(x^{2}+u_{1} x+u_{0}, v_{1} x+v_{0}\right)$.

Let $D_{1}=\left(U_{1}, V_{1}\right), D_{2}=\left(U_{2}, V_{2}\right) \in \mathcal{J}_{C}\left(\mathbb{F}_{2^{m}}\right)$ be reduced divisors. The computational cost of a divisor class doubling $D_{3}=\left(U_{3}, V_{3}\right)=2 D_{1}$ and a divisor class addition $D_{3}=D_{1}+D_{2}$ depends on the conditions that $D_{1}, D_{2}$ and $D_{3}$ satisfy. We list some conditions as follows:

DBL $w\left(D_{1}\right)=2, w\left(D_{3}\right)=2, \operatorname{gcd}\left(h, U_{1}\right)=1$,

ADD $w\left(D_{1}\right)=2, w\left(D_{2}\right)=2, w\left(D_{3}\right)=2, \operatorname{gcd}\left(U_{1}, U_{2}\right)=1$,

TDBL $w\left(D_{1}\right)=1, w\left(D_{3}\right)=2, \operatorname{gcd}\left(h, U_{1}\right)=1$,

TADD $\quad w\left(D_{1}\right)=2, w\left(D_{2}\right)=1, w\left(D_{3}\right)=2, \operatorname{gcd}\left(U_{1}, U_{2}\right)=1$.

${ }^{1}$ A theta divisor is called a degenerate divisor in 9 . 
DBL and ADD correspond to so-called most frequent cases of a divisor class doubling and an addition, respectively. TDBL denotes the doubling of a theta divisor. TADD denotes the addition of a general divisor and a theta divisor.

The computational cost of TDBL and TADD is smaller than that of DBL and ADD, respectively, because of simple representation of a theta divisor. We summarize their cost in Table 1] $M, S$, and $I$ denote the required time of multiplication, squaring, and inversion, respectively.

Table 1. Cost of group operations (genus $2, C / \mathbb{F}_{2^{m}}$ )

\begin{tabular}{l|c}
\hline \hline Group operations & Cost \\
\hline \hline DBL [13] & $1 I+22 M+5 S$ \\
ADD [13] & $1 I+22 M+3 S$ \\
\hline TDBL [8] & $1 I+5 M+2 S$ \\
TADD [13] & $1 I+10 M+1 S$ \\
\hline \hline
\end{tabular}

\section{Scalar Multiplication on HECC}

\subsection{Double-and-Add-Always Method}

In order to construct HECC, it is necessary to compute scalar multiplication $d D$, where $d$ is a non-negative integer and $D$ is a reduced divisor. Let $d=$ $\left(d_{n-1} \cdots d_{0}\right)_{2}$ be the binary representation of $d$, where $d_{n-1}=1$. The most standard SPA-resistant method to compute $d D$ is called the Double-and-AddAlways method (DAA), shown in Algorithm 1

Algorithm 1. Double-and-add-always Method (DAA)

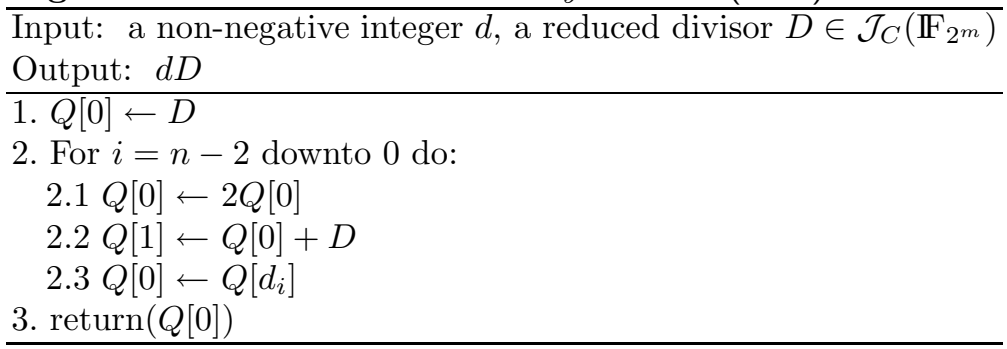

In step 2.1 and step 2.2, a divisor class doubling and a divisor class addition are computed, respectively. If the input divisor $D$ is a general divisor, a doubling in step 2.1 corresponds to DBL and an addition in step 2.2 corresponds to ADD with very high probability. On the other hand, if $D$ is a theta divisor, ADD is replaced by TADD. From Table 1, we estimate the computational cost of scalar multiplication $d D$ of both a general divisor and a theta divisor. DAA of a General Divisor, which we call DAA_GD, takes $(1 I+22 M+3 S)+(1 I+22 M+5 S)=$ $2 I+44 M+8 S$ per bit of the scalar $d$. On the other hand, DAA of a Theta 
Divisor, which we call DAA_TD, takes only $(1 I+10 M+1 S)+(1 I+22 M+5 S)=$ $2 I+32 M+6 S$ per bit of $d[8$. Therefore the idea choosing a theta divisor as the input divisor can achieve about $20 \%$ improvement of efficiency under $I=8 \mathrm{M}$ and $S=0.1 M$. Its cryptographic application, however, is limited; even if we choose a theta divisor as the base point of HECC, we must often compute scalar multiplication of a general divisor.

DAA repeats the identical sequence of a doubling and an addition by inserting dummy additions for $d_{i}=0$ in step 2.2. Thus, an SPA attacker cannot guess any bit information of $d$. The insertion of dummy operations, however, causes the possibility of fault-based attacks.

\subsection{Simultaneous Scalar Multiplication}

Let us consider the sum of scalar multiplication $k D_{1}$ and $l D_{2}$, where $k, l$ are nonnegative integers and $D_{1}, D_{2}$ are reduced divisors. It is necessary to compute the sum $k D_{1}+l D_{2}$ in HEC-DSA signature verification. The efficient method to compute $k D_{1}+l D_{2}$ is known as Shamir's method. This method computes $k D_{1}+l D_{2}$ simultaneously instead of computing $k D_{1}$ and $l D_{2}$ independently, so that it is called simultaneous scalar multiplication.

Let $k=\left(k_{n-1} \cdots k_{0}\right)_{2}$ and $l=\left(l_{n-1} \cdots l_{0}\right)_{2}$ be the binary representations of $k$ and $l$, respectively, where $k_{n-1}=1$ or $l_{n-1}=1$. The simultaneous scalar multiplication $k D_{1}+l D_{2}$ is shown in Algorithm 2.

\section{Algorithm 2. Simultaneous Scalar Multiplication}

Input: non-negative integers $k, l$ and reduced divisors $D_{1}, D_{2}$

Output: $k D_{1}+l D_{2}$

1. (pre-computation) compute $D_{1}+D_{2}$

2. $Q \leftarrow k_{n-1} D_{1}+l_{n-1} D_{2}$

3. For $i=n-2$ downto 0 do:

3.1. $Q \leftarrow 2 Q$

3.2. if $\left(k_{i}, l_{i}\right) \neq(0,0)$ then

$$
T \leftarrow Q+\left(k_{i} D_{1}+l_{i} D_{2}\right)
$$

4. return $Q$

Algorithm 2 can reduce the number of doublings to half compared to computing $k D_{1}$ and $l D_{2}$ separately. Furthermore we define the following terms in order to evaluate the number of additions.

Definition 1. Let $\left\langle k_{n-1} \cdots k_{0}\right\rangle$ and $\left\langle l_{n-1} \cdots l_{0}\right\rangle$ be signed binary representations of non-negative integers $k$ and $l$, respectively. The number of $i(0 \leq i \leq n-1)$ satisfying $\left(k_{i}, l_{i}\right) \neq(0,0)$ is called Joint Hamming Weight of $(k, l)$.

Definition 2. Let $\left\langle k_{n-1} \cdots k_{0}\right\rangle$ and $\left\langle l_{n-1} \cdots l_{0}\right\rangle$ be signed binary representations of non-negative integers $k$ and $l$, respectively. The ratio of Joint Hamming Weight of $(k, l)$ to $n$ is called Joint Hamming Density. 
The average Joint Hamming Density is $3 / 4$ for the binary representations of $k$ and $l$. As a result, the number of additions required in Algorithm 2 is about $3 n / 4$ on average.

In order to speed up simultaneous scalar multiplication, Solinas gave the efficient signed binary representation of two non-negative integers [17. The representation is called Joint Sparse Form (JSF), and any pair of non-negative integers has unique JSF. The average Joint Hamming Density of JSF is $1 / 2$, so that simultaneous scalar multiplication with JSF can reduce the number of additions to about $n / 2$ on average.

\section{Divisor Decomposition Technique}

In this section we propose a novel technique called Divisor Decomposition Technique (DDT).

We now consider a general divisor $D=\left(x^{2}+u_{1} x+u_{0}, v_{1} x+v_{0}\right)$, where $u_{i}, v_{i} \in$ $\mathbb{F}_{2^{m}}$ for $i=0,1$. $D$ can be decomposed into two theta divisors $D_{1}=\left(x+x_{1}, y_{1}\right)$ and $D_{2}=\left(x+x_{2}, y_{2}\right)$ if $x^{2}+u_{1} x+u_{0}$ is factored to $\left(x+x_{1}\right)\left(x+x_{2}\right)$ over $\mathbb{F}_{2^{m}}$. It depends on only the reducibility of $x^{2}+u_{1} x+u_{0}$ over $\mathbb{F}_{2^{m}}$ whether $D$ can be decomposed or not. Consequently, $\operatorname{Tr}\left(u_{0} / u_{1}^{2}\right)=0$ is the only condition of divisor decomposition, where $\operatorname{Tr}(c)$ is trace of $c \in \mathbb{F}_{2^{m}}$. We show the procedure of DDT in Algorithm 3, where $\operatorname{Hr}(c)$ is half-trace of $c$. $T$ and $H$ denote the computational cost of trace and half-trace, respectively.

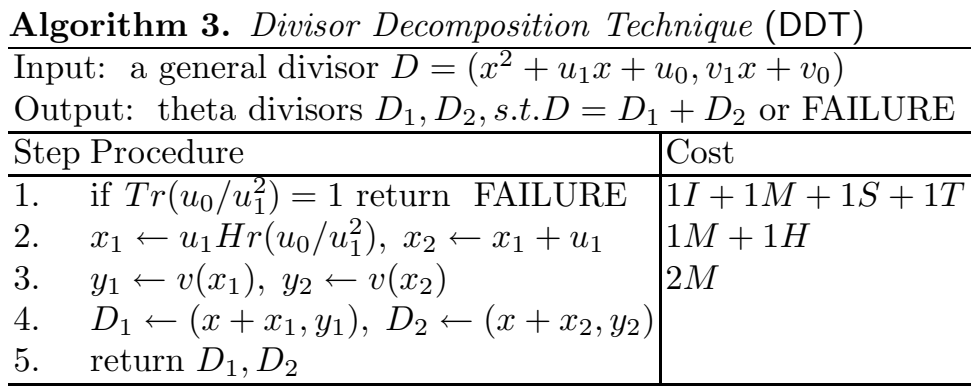

The question we have to ask here is whether DDT contributes to the efficiency of scalar multiplication $d D$. As we have seen, each scalar multiplication $d D_{1}, d D_{2}$ is faster than $d D$. The direct computation of $d D_{1}+d D_{2}$, however, is slower than scalar multiplication $d D$ because Table 1 shows that TADD is not twice as fast as ADD.

\section{Joint Regular Form}

In this section we propose the other novel technique called Joint Regular Form (JRF).

We define a signed binary representation for a pair of non-negative integers as follows. 
Definition 3. Let $\left\langle k_{n-1} \cdots k_{0}\right\rangle$ and $\left\langle l_{n-1} \cdots l_{0}\right\rangle$ be signed binary representations of $k$ and $l$, respectively, satisfying $k+l \equiv 1(\bmod 2) .\left\langle k_{n-1} \cdots k_{0}\right\rangle$ and $\left\langle l_{n-1} \cdots l_{0}\right\rangle$ is called Joint Regular Form (JRF) of $(k, l)$, if $k_{i}$ and $l_{i}$ satisfy $k_{i}+l_{i}= \pm 1$, that $i s,\left(k_{i}, l_{i}\right)=(0, \pm 1)$ or $( \pm 1,0)$ for any $i$.

Example 1. JRF of $(52,39)$ is represented as follows, where $\overline{1}$ denotes -1 .

$$
\begin{aligned}
& 52=\left\langle\begin{array}{lllllll}
1 & 0 & \overline{1} & 0 & 1 & 0 & 0
\end{array}\right\rangle \\
& 39=\left\langle\begin{array}{lllllll}
0 & 1 & 0 & 1 & 0 & \overline{1} & 1
\end{array}\right\rangle
\end{aligned}
$$

The Joint Hamming Density of JRF is always 1. JRF has the following properties:

- A pair of non-negative integers $(k, l)$ satisfying $k+l \equiv 1(\bmod 2)$ has a JRF.

- JRF of a certain length is unique.

We first prove the uniqueness of JRF.

Theorem 1. A pair $(k, l)$ of non-negative integers has at most one Joint Regular Form of a certain length.

Proof. Assume that there are two distinct JRFs of length $n$ as

$$
\begin{aligned}
k & =\left\langle k_{n-1} \cdots k_{0}\right\rangle=\left\langle k_{n-1}^{\prime} \cdots k_{0}^{\prime}\right\rangle \\
l & =\left\langle l_{n-1} \cdots l_{0}\right\rangle=\left\langle l_{n-1}^{\prime} \cdots l_{0}^{\prime}\right\rangle .
\end{aligned}
$$

Let $j$ be the minimal value satisfying $k_{i} \neq k_{i}^{\prime}$ or $l_{i} \neq l_{i}^{\prime}$, and

$$
\begin{aligned}
& s=\left\langle k_{n-1} \cdots k_{j}\right\rangle=\left\langle k_{n-1}^{\prime} \cdots k_{j}^{\prime}\right\rangle \\
& t=\left\langle l_{n-1} \cdots l_{j}\right\rangle=\left\langle l_{n-1}^{\prime} \cdots l_{j}^{\prime}\right\rangle .
\end{aligned}
$$

We may assume that $k_{j} \neq k_{j}^{\prime}$ by exchanging $k$ and $l$ if necessary. It follows that $k_{j}$ and $k_{j}^{\prime}$ have value 1 and -1 . We assume that $k_{j}=1$ and $k_{j}^{\prime}=-1$ without loss of generality. By the definition of JRF, $l_{j}=0$ and $l_{j}^{\prime}=0$.

Suppose that $s \equiv 1(\bmod 4) \cdot k_{j+1}=0$ and $k_{j+1}^{\prime}= \pm 1$ since $k_{j}=1$ and $k_{j}^{\prime}=-1$. It follows that $l_{j+1}= \pm 1$ and $l_{j+1}^{\prime}=0$. The former indicates that $t \equiv 2(\bmod 4)$ and the latter indicates that $t \equiv 0(\bmod 4)$. This contradiction shows that the initial assumption must be wrong. Supposing $s \equiv 3(\bmod 4)$, the similar contradiction occurs.

The most straightforward way to prove the existence of JRF for any pair of nonnegative integers $(k, l)$ satisfying $k+l \equiv 1(\bmod 2)$ is to present an algorithm for constructing it. We explain how to construct JRF from the least significant bit. Let $\left(k_{n-1} \cdots k_{0}\right)_{2}$ and $\left(l_{n-1} \cdots l_{0}\right)_{2}$ be the binary representations of $k$ and $l$, respectively. Firstly, $\left(k_{0}, l_{0}\right)=(0,1)$ or $(1,0)$ by $k+l \equiv 1(\bmod 2)$.

Next, we notice $\left(k_{1}, l_{1}\right)$. If $\left(k_{1}, l_{1}\right)=(0,0)$, either of the following transformations is carried out according to $\left(k_{0}, l_{0}\right)$. 


\begin{tabular}{|c|c|c|c|c|c|c|c|c|}
\hline$k_{1}$ & $k_{0}$ & & $k_{1}$ & $k_{0}$ & $k_{1}$ & $k_{0}$ & & $k_{1}$ \\
\hline 0 & 1 & & 1 & $\overline{1}$ & 0 & 0 & & 0 \\
\hline 0 & 0 & $\Rightarrow$ & 0 & 0 & 0 & 1 & $\Rightarrow$ & 1 \\
\hline & $l_{0}$ & & $l_{1}$ & $l_{0}$ & $l_{1}$ & $l_{0}$ & & $l_{1}$ \\
\hline
\end{tabular}

If $\left(k_{1}, l_{1}\right)=(1,1)$, one performs either of the following transformations according to $\left(k_{0}, l_{0}\right)$.

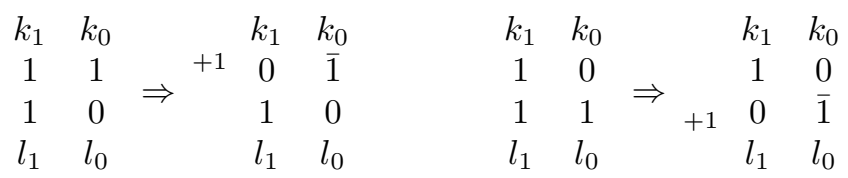

+1 means 1 is carried over to either $\left(k_{n-1} \cdots k_{2}\right)_{2}$ or $\left(l_{n-1} \cdots l_{2}\right)_{2}$. If $\left(k_{1}, l_{1}\right)=$ $(0,1)$ or $(1,0)$, one needs no transformation. In all cases of $\left(k_{1}, l_{1}\right)$, it is possible to satisfy the following conditions: $\left(k_{0}, l_{0}\right)=(0, \pm 1)$ or $( \pm 1,0)$, and $\left(k_{1}, l_{1}\right)=(0,1)$ or $(1,0)$.

By applying this transformation from the least significant bit, we construct the signed binary representations $\left\langle k_{n-1} \cdots k_{0}\right\rangle$ and $\left\langle l_{n-1} \cdots l_{0}\right\rangle$ satisfying $\left(k_{i}, l_{i}\right)=$ $(0, \pm 1)$ or $( \pm 1,0)$ for any $i$. The detailed algorithm is shown in Algorithm 4 .

\section{Algorithm 4. Joint Regular Form (JRF)}

Input: a pair of non-negative integers $(k, l)$ s.t. $k+l \equiv 1(\bmod 2)$

Output: JRF of $(k, l):\left\langle k_{n-1} \cdots k_{0}\right\rangle,\left\langle l_{n-1} \cdots l_{0}\right\rangle$

1. $i \leftarrow 0, s \leftarrow k, t \leftarrow l$,

2. while $s>0$ or $t>0$ do:

2.1. $k_{i}=s \bmod 2, l_{i}=t \bmod 2$

2.2. if $\left(k_{i}, l_{i}\right)=(0,0)$ then

$$
k_{i} \leftarrow k_{i-1}, k_{i-1} \leftarrow-k_{i-1}, l_{i} \leftarrow l_{i-1}, l_{i-1} \leftarrow-l_{i-1}, s \leftarrow s / 2, t \leftarrow t / 2
$$

else if $\left(k_{i}, l_{i}\right)=(1,1)$ then

$$
\begin{aligned}
& k_{i} \leftarrow 1-k_{i-1}, k_{i-1} \leftarrow-k_{i-1}, l_{i} \leftarrow 1-l_{i-1}, l_{i-1} \leftarrow-l_{i-1}, \\
& s \leftarrow\left(s-2 k_{i}+1\right) / 2, t \leftarrow\left(t-2 l_{i}+1\right) / 2
\end{aligned}
$$

else then

2.3. $i \leftarrow i+1$

$$
s \leftarrow\left(s-k_{i}\right) / 2, t \leftarrow\left(t-l_{i}\right) / 2
$$

3. $n \leftarrow i$

4. return $\left\langle k_{n-1} \cdots k_{0}\right\rangle$ and $\left\langle l_{n-1} \cdots l_{0}\right\rangle$

If we apply JRF of $(k, l)$ to the simultaneous scalar multiplication $k D_{1}+l D_{2}$, we always compute a divisor class doubling and an addition of $\pm D_{1}$ or $\pm D_{2}$ per bit of $(k, l)$. Consequently, we achieves the SPA-resistant simultaneous scalar multiplication without any dummy operation and pre-computation $D_{1} \pm D_{2}$.

Remark 1. The advantages of simultaneous scalar multiplication with JRF are useful to not only HECC but also ECC. Lim-Lee method [14], GLV method [6], and BRIP [15] seem to be nice applications of JRF. 


\section{Combination of DDT and JRF}

We show that the combination of DDT and JRF achieves efficient and secure scalar multiplication of a general divisor.

Suppose that a general divisor $D$ can be decomposed into two theta divisors $D_{1}$ and $D_{2}$ as $D=D_{1}+D_{2}$. We compute $\left(d D_{1}+d^{\prime} D_{2}\right)-D_{2}$ instead of the scalar multiplication $d D$, where $d^{\prime}=d+1$. JRF of $\left(d, d^{\prime}\right)$ is applied to the simultaneous scalar multiplication $d D_{1}+d^{\prime} D_{2}$. As we have discussed above, $d D_{1}+d^{\prime} D_{2}$ with JRF computes a divisor class doubling and an addition of a theta divisor $\pm D_{1}$ or $\pm D_{2}$ per bit of $\left(d, d^{\prime}\right)$.

Indeed, JRF of $\left(d, d^{\prime}\right),\left\langle d_{n} \cdots d_{0}\right\rangle$ and $\left\langle d_{n}^{\prime} \cdots d_{0}^{\prime}\right\rangle$, can be represented very easily without Algorithm 4 as follows:

1. Let $\left\langle d_{n-1} \cdots d_{0}\right\rangle$ be the binary representation of $d$.

2. $d_{i}^{\prime}=d_{i}-1$ for $0 \leq i \leq n-1$.

3. Append $d_{n}=0$ and $d_{n}^{\prime}=1$.

The validity of this representation is clearly shown by $d=\sum_{i=0}^{n-1} d_{i} 2^{i}$ and $d^{\prime}=$ $d+1=2^{n}+\sum_{i=0}^{n-1}\left(d_{i}-1\right) 2^{i}$. For example, JRF of $(53,54)$ is represented as $53=\langle 0110101\rangle$ and $54=\langle 100 \overline{1} 0 \overline{1} 0\rangle$.

We present the detailed algorithm for theta divisors $D_{1}$ and $D_{2}$ in Algorithm 5 . Obviously Algorithm 5 always computes a divisor class doubling and an addition of a theta divisor whether $d_{i}=0$ or 1 . Therefore, an SPA-attacker cannot guess any bit information of $d$.

\section{Algorithm 5. Simultaneous Scalar Multiplication with JRF (SimJRF)}

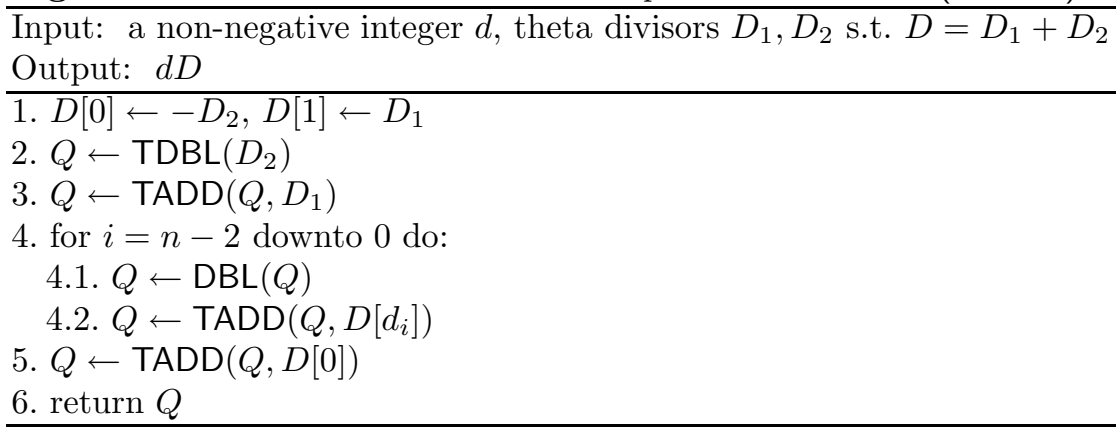

As described in Section 4, a general divisor $D$ cannot be always decomposed to two theta divisors; $D$ can be decomposed only if $x^{2}+u_{1} x+u_{0}$ is reducible over $\mathbb{F}_{2^{m}}$, where $D=\left(x^{2}+u_{1} x+u_{0}, v_{1} x+v_{0}\right)$. In order to apply DDT to any general divisor, we utilize a divisor class doubling (DBL) and its inverse operation, that is, a divisor class halving (HLV) [10. If $D$ cannot be decomposed, one repeats $i$ times until $D^{\prime}=2^{i} D$ can be decomposed by $D^{\prime}=D_{1}^{\prime}+D_{2}^{\prime}$. After computing $d D^{\prime}$ using SimJRF, one then repeats a divisor class halving $i$ times by $d D=1 / 2^{i}\left(d D^{\prime}\right)$. We summarize our efficient and secure scalar multiplication algorithm for a general divisor in Algorithm 6 . 
Algorithm 6. DDT and SimJRF (DDT+SimJRF)

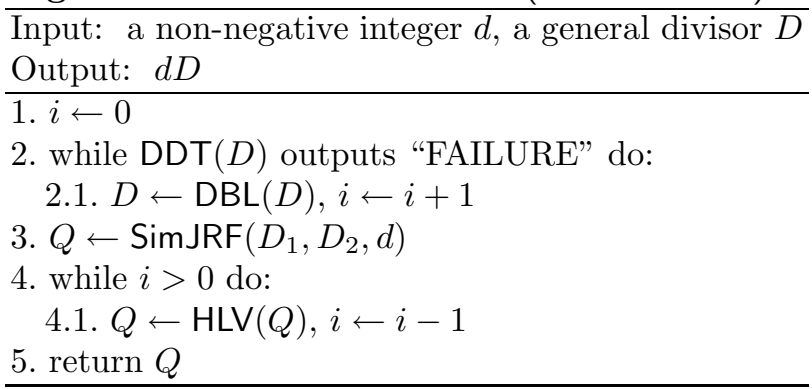

The iteration count $i$ becomes 1 on average since DDT returns "FAILURE" in probability of about $1 / 2$. Accordingly, we require two DDT and one DBL in step 2, and one HLV in step 4.

Remark 2. In HEC ElGamal-type decryption, a receiver $A$ needs to compute $s_{A} D$, where $s_{A}$ is $A$ 's secret key and $D$ is a random divisor. In the case, instead of the operations DBL and HLV, we can utilize an addition of the base point $G$ and a subtraction of $s_{A} G$, where $s_{A} G$ is $A$ 's public key.

\section{Computational Efficiency}

We estimate the computational cost of DDT+SimJRF proposed in Algorithm 6 and compare it to that of the Double-and-Add-Always method of a General Divisor (DAA_GD) and the Double-and-Add-Always method of a Theta Divisor (DAA_TD). The cost of divisor doublings and additions is referred to Table 1]. As we have mentioned in Section 3.1, the cost of DAA_GD and DAA_TD is $(n-$ $1)(2 I+44 M+8 S)$ and $(n-2)(2 I+32 M+6 S)+2 I+28 M+3 S$, respectively, where $n$ is the bit length of $d$.

In order to estimate the cost of DDT+SimJRF in Algorithm 6, we evaluate the cost required in divisor decomposition step (step 2), SimJRF (step 3), and compensation step (step 4) as following.

divisor decomposition step. According to our analysis in Section 6, we require two DDT, one of which returns "FAILURE", and one DBL on average. The estimated cost is $2(1 I+1 M+1 S+1 T)+(3 M+1 H)+(1 I+22 M+5 S)=$ $3 I+27 M+7 S+H+2 T$.

SimJRF. We estimate the cost of Sim JRF through Algorithm 5, In step 1, the inverse of a theta divisor $D_{2}=\left(x+x_{2}, y_{2}\right),-D_{2}=\left(x+x_{2}, y_{2}+h\left(x_{0}\right)\right)$, is computed, which corresponds to $1 M$. Step 2 and 3 are the main procedures of Sim JRF. We then require one TDBL, $(n-1) \mathrm{DBL}$, and $n$ TADD, which correspond to $(1 I+5 M+2 S)+(n-1)(1 I+22 M+5 S)+n(1 I+10 M+1 S)=(n-1)(2 I+$ $32 M+6 S)+2 I+15 M+3 S$. Step 4 requires $1 I+10 M+1 S$. As a result, the total cost of Sim JRF is estimated to be $(n-1)(2 I+32 M+6 S)+3 I+26 M+4 S$. 
compensation step. We require one $\mathrm{HLV}$ as we have shown in Section 6] According to [10], the cost of HLV is $1 I+19.5 M+2 S+2.5 S R+2 H+2 T$, where $S R$ denotes the cost of square root over $\mathbb{F}_{2^{m}}$.

Consequently, the cost of DDT+SimJRF is estimated to be $n(2 I+32 M+$ $6 S)+5 I+40.5 M+7 S+2.5 S R+3 H+4 T$ in total.

Suppose that the bit length of $d$ is 160, that is, $n=160$. According to [510], we may assume that the following ratios of field operations to multiplication are satisfied: $I=8 M, S=0.1 M, S R=0.5 M, H=0.6 M$, and $T=0$.

Table 2 summarizes the comparison of DAA_GD, DAA_TD, and DDT+SimJRF. The column 'Divisor' indicates whether each method computes scalar multiplication of a general divisor or a theta divisor; the column 'Dummy' indicates whether each method uses any dummy operation or not. The proposed method DDT+SimJRF is $18.7 \%$ faster than DAA_GD and eliminates any dummy operation. DDT+SimJRF requires no more than $1.8 \%$ increase of computational cost compared to DAA_TD that can be used only in the limited case.

Table 2. Comparison of scalar multiplication (160bit)

\begin{tabular}{l|c|c|lc}
\hline Method & Divisor & Dummy & Cost & \\
\hline DAA_GD & general & use & $318 I+6996 M+1272 S$ & $(9667.2 M)$ \\
DAA_TD & theta & use & $318 I+5084 M+951 S$ & $(7723.1 M)$ \\
DDT+SimJRF & general & NOT use & $325 I+5160.5 M+967 S$ & \\
& & & \multicolumn{3}{|c}{$+2.5 S R+3 H+4 T$} & $(7860.3 M)$ \\
\hline
\end{tabular}

\section{Conclusion and Further Work}

In this paper, efficient and secure scalar multiplication of a general divisor for genus 2 HECC over $\mathbb{F}_{2^{m}}$ is proposed through Divisor Decomposition Technique (DDT) and Joint Regular Form (JRF). The proposed method achieves both about $19 \%$ improvement of efficiency compared to the double-and-add-always method and SPA resistance without any dummy operation.

It must be emphasized that the strategy of the proposed method is applicable to not only genus $2 \mathrm{HECC}$ over $\mathbb{F}_{2^{m}}$. For genus $3 \mathrm{HECC}$, a general divisor whose weight is 3 might be decomposed into either three theta divisors of weight 1 or two theta divisors of weight 2 . In the former case, we must generalize the concept of JRF to three non-negative integers. In the latter case, we need to develop efficient DDT in which a weight 3 divisor is decomposed into two weight 2 divisors.

\section{References}

1. D.G. Cantor, "Computing in the Jacobian of a Hyperelliptic Curve", Mathematics of Computation, 48, 177, pp.95-101, 1987.

2. H. Cohen, G. Frey, R. Avanzi, C. Doche, T. Lange, K. Nguyen, and F. Vercauteren, Handbook of Elliptic Curve and Hyperelliptic Curve Cryptography, Chapman \& Hall, 2005. 
3. J.-S. Coron, "Resistance against Differential Power Analysis for Elliptic Curve Cryptosystems", Cryptographic Hardware and Embedded Systems - CHES '99, LNCS 1717, pp.292-302, Springer-Verlag, 1999.

4. S. Duquesne, "Montgomery Scalar Multiplication for Genus 2 Curves", Algorithmic Number Theory - ANTS VI, LNCS 3076, pp.153-168, Springer-Verlag, 2004.

5. K. Fong, D. Hankerson, J. López and A. Menezes, "Field inversion and point halving revised," Technical Report CORR 2003-81, 2003. http://www.cacr.math. uwaterloo.ca/techreports/2003/corr2003-18.pdf

6. R.P. Gallant, R.J. Lambert, and S.A. Vanstone, "Faster Point Multiplication on Elliptic Curves with Efficient Endomorphisms", Advances in Cryptology - CRYPTO 2001, LNCS 2139, pp.190-200, Springer-Verlag, 2001.

7. R. Harley, "Adding.txt, Doubling.c", 2000. http://cristal.inria.fr/ harley/hyper/

8. M. Katagi, T. Akishita, I. Kitamura, and T. Takagi, "Efficient Hyperelliptic Curve Cryptosystems Using Theta Divisors", IEICE Trans. Fundamentals, vol.E89-A, no.1, pp.151-160, 2006.

9. M. Katagi, I. Kitamura, T. Akishita, and T. Takagi, "Novel Efficient Implementations of Hyperelliptic Curve Cryptosystems Using Degenerate Divisors", Information Security Application s - WISA 2004, LNCS 3325, pp.345-359, Springer-Verlag, 2004 .

10. I. Kitamura, M. Katagi, and T. Takagi, "A Complete Divisor Class Halving Algorithm for Hyperelliptic Curve Cryptosystems of Genus Two", Information Security and Privacy - ACISP 2005, LNCS 3674, pp.146-157, Springer-Verlag, 2005.

11. N. Koblitz, "Hyperelliptic Cryptosystems", Journal of Cryptology, vol.1, pp. 139-150, Springer-Verlag, 1989.

12. S. Lang, "Abelian Varieties", Springer-Verlag, 1983.

13. T. Lange, "Formulae for Arithmetic on Genus 2 Hyperelliptic Curves", Applicable Algebra in Engineering, Communication and Computing, vol.15, pp.295-328, Springer-Verlag, 2005.

14. C.H. Lim and P.J. Lee, "More Flexible Exponentiation with Precomputation", Advances in Cryptology - CRYPTO '94, LNCS 839, pp.95-107, Springer-Verlag, 1994.

15. H. Mamiya, A. Miyaji, and H. Morimoto, "Efficient Countermeasure against RPA, DPA, and SPA", Cryptographic Hardware and Embedded Systems - CHES 2004, LNCS 3156, pp.343-356, Springer-Verlag, 2004.

16. D. Mumford, Tata Lectures on Theta II, Progress in Mathematics 43, Birkhäuser, 1984.

17. J.A. Solinas, "Low-Weight Binary Representations for Pairs of Integers", Technical Report CORR 2001-41, 2001. http://www.cacr.math.uwaterloo.ca/ techreports/2001/corr2001-41.ps 